\title{
4. Comunicación transmedia y ciudad. El caso de La Candelaria de Bogotá, Colombia
}

\author{
Stefania Ramírez López ${ }^{1}$
}

\section{Resumen}

Ciudades como Barcelona, Nueva York, Niza, Londres, entre otras, son catalogadas como las más innovadoras a nivel mundial de acuerdo con el reporte de Ciudades en Movimiento 2018, del IESE Cities in Motion Strategies (2018), e incluyen en su Plan de Desarrollo tecnologías de la información que buscan enriquecer la calidad de vida de sus habitantes y turistas. Sin embargo, existen ciudades que pocas veces hacen uso de éstas para mejorar la experiencia a la hora de conocer la historia. Esta investigación busca ir más allá, y pretende lograr que por medio de la comunicación transmedia e hipermedia se mejore la experiencia del usuario, sea habitante de la ciudad o visitante, para que se pueda descubrir de manera atractiva su patrimonio material e inmaterial, especialmente el segundo, y donde a su vez exista interactividad e interacción tanto física como virtual a partir de un dispositivo móvil. El presente trabajo tendrá como objeto de estudio la ciudad de Bogotá, Colombia, específicamente la localidad La Candelaria.

Palabras clave: Comunicación, Hipermedia, Patrimonio Material e Inmaterial, Tecnología, Transmedia.

1. Comunicadora social y periodista de la Universidad Externado de Colombia, magíster en Comunicación Audiovisual para la Era Digtalde la Universidad Complutense de Madrid y doctorando en Comunicación Audiovisual, Publicidad y Relaciones Públicas de la misma universidad. Docente universitaria e investigadora del Programa de Comunicación y Gestión de la Información del Politécnico Grancolombiano. Miembro del grupo Museum $1+D+c^{\prime}$ Laboratorio de cultura digital y museografía hipermedia en la Universidad Complutense de Madrid.Correo electrónico:sramirezl@poligran.edu.co 


\section{Transmedia Communication and the City.}

\section{The case of La Candelaria in Bogotá, Colombia.}

\section{Abstract}

Cities like Barcelona, New York, Nice, London, among others, are classified as the most innovative in the world according to Cities in Motion Strategies (2018) report. They include in their Development Plan information technology, seeking to enhance the quality of life for residents and tourists. However, there are cities that rarely make use of these to improve the experience when it comes to knowing the history. This research seeks to go beyond, what we want to achieve is to improve the user experience through transmedia communication and hypermedia, whether you are a resident or a visitor of the city, in an attractive way to discover the tangible and intangible heritage, and also with interactivity and interaction in a physical and virtual way from a mobile device. This investigation will study the city of Bogotá, Colombia, specifically the locality of La Candelaria.

Keywords: Communication, Hypermedia, Tangible and Intangible Cultural Heritage, Technology, Transmedia.

\section{Introducción}

lo largo de los últimos años se ha podido apreciar que las ciudades han tenido grandes cambios, haciendo uso de las Tecnologías de la Información y Comunicación (TIC) y de los procesos de innovación en la comunicación, aspirando así a convertirse en lugares funcionales, modernos y sostenibles.

Por ello, una ciudad debe apuntar a desarrollar las TIC para su bienestar, con el fin de rescatar y exponer su historia, generando nuevas posibilidades de visibilidad a las personas para que se apropien de la ciudad e interactúen con la misma, atrayendo así más visitantes. Una ciudad donde no solamente se haga uso de lo material como los monumentos, sino que a partir de lo inmaterial se pueda crear una experiencia única. Por eso nace esta investigación, que pretende descubrir cómo se percibe a La Candelaria de Bogotá, y cómo se aprecia y disfruta de su recorrido histórico por parte de las personas, base para que posteriormente, en otra investigación, se puedan estudiar, analizar y desarrollar estrategias para que las ciudades no dejen de lado su pasado, haciendo uso de los nuevos medios de comunicación digitales convergentes, la interacción, la interactividad y de las nuevas formas de producción audiovisual, para que al final se pueda conservar y comunicar su patrimonio material e inmaterial. Con base en esto, se plantea el principal objetivo de esta investigación, el cual es identificar y presentar las tecnologías creadas para desarrollar y gestionar la información TIC, y la importancia de la interactividad y la interacción, no solamente física sino también personal, para así difundir de manera atractiva la información sobre el patrimonio material e inmaterial de la localidad.

De acuerdo con Isidro Moreno (2014, p. 90) la "interacción se refiere a acciones dinámicas sin mediación tecnológica, y la interactividad refleja esas acciones dinámicas valiéndose de la tecnología". Por otro lado, Freitas y Castro (2010, p. 25) hacen mención en su texto Narrativas audiovisuales y tecnologías interactivas, a que la interactividad es un concepto indispensable a la hora de la NT, visto que "depende de las sensaciones 
despertadas por el contenido y las posibilidades de manipulación del objeto ofertado al público, proporcionados por el lenguaje y por el soporte utilizado como medio de expresión".

Este proyecto se realiza con el fin de recopilar información, siendo esta una primera aproximación al tema de estudio puesto que actualmente existe poco desarrollo del tema, algo que sin duda es una oportunidad de innovación para el beneficio no solo de la localidad, sino también de la ciudad de Bogotá. Para iniciar la presente investigación es necesario hablar sobre La Candelaria, también conocida como el centro histórico de la capital, que, de acuerdo con el Departamento de Planeación Distrital; es la localidad número 17 de la ciudad de Bogotá, cuya población es 23.625 habitantes permanentes, con una extensión de 183.89 hectáreas, ubicada en el sector centro-oriental, con un total de 2.364 predios, de los cuales 54 se encuentran declarados como Bienes de Interés Cultural del Orden Nacional y 1.608 son predios de conservación arquitectónica (Alcaldía Mayor de Bogotá. Secretaría General).

\section{Historia fundamental de La Candelaria}

Jaime Umaña Díaz rescata en su artículo "La raíz de nuestra historia - La Candelaria: ayer y hoy" la descripción narrada por el historiador de la conquista Don Juan de Castellanos que describe el pensamiento con el que fue creada la ciudad de Bogotá, especialmente La Candelaria, donde las leyes indígenas construían las ciudades teniendo en cuenta la "trama de retícula" con forma de damero, que permitía la edificación en un lugar con la suficiente altura para que las aguas corrieran tranquilamente sin que invadieran las plazas y calles, además de ser en piedra, leña y buenos aires (2008, p. 23,24).

De acuerdo con la Alcaldía Mayor de Bogotá no existe acta de fundación, más la fecha aceptada fue el 6 de agosto de 1538 por Gonzalo Jiménez de Quesada, y estratégicamente se realizó en Teusaquillo, actualmente el Chorro de Quevedo en la Candelaria, ya que se encuentra:

En medio de dos ríos, el San Francisco y el San Agustín, protegida en su espalda por el cerro de Monserrate y con una perspectiva clara sobre la Sabana, de particular importancia en caso de ataques. Esa localización le permitió a Santafé primero y a Bogotá después, protegerse de las guerras que tanto amenazaron su tranquilidad (Secretaría Distrital de Planeación, 2009, p. 9).

A partir de ese momento han pasado diversos acontecimientos en la historia de La Candelaria, convirtiéndose en una zona llena de hechos históricos, donde cada calle, casa, plaza y esquina cuentan vivencias que conforman lo que hoy en día es la ciudad.

\section{Comunicación transmedia y ciudad}

A finales de los años 90 se empieza a construir un pensamiento acerca de las estructuras físicas de la ciudad como espacio para el desarrollo de una red electrónica y de las tecnologías digitales, de acuerdo con Toyo Ito en 1999, donde se buscaba explicar la hibridación como "el espacio requerido por ambos cuerpos que se llena con transparencia y homogeneidad... se trata de un espacio transparente de extensión infinita, pero era un espacio visible al que se podría dar forma" (2006, p. 19).

Hoy en día se habla de Smart City o ciudad inteligente, un concepto cuyo objetivo es el de suministrar a la ciudad "una infraestructura que intente garantizar un desarrollo sostenible, un incremento de la calidad de vida de sus ciudadanos, una mayor eficiencia de sus recursos humanos y energéticos. Resumidamente, se trata de usar la tecnología en beneficio de la población" (Bustillo y Rodríguez, 2015, p. 258).

Experiencias únicas, interacción e interactividad, son conceptos que se buscan aplicar en la localidad de La Candelaria, tratando de experimentar con los espacios físicos, llevando a cabo una comunicación transmedia, como menciona Russell:

Lo que antes era exclusivo de los constructores, arquitectos e ingenieros, hoy está en manos de todos: la capacidad de dar forma y organizar el espacio y el mundo real... cubriendo todo con una capa invisible de anotaciones; la información textual, visual y sonora está disponible cuando te acercas, cuando el contexto lo indique 0 cuando la pides (1999, pp. 4 y 30)

Ahora bien, existen muchas ciudades en el mundo pioneras en la creación de actividades culturales innovadoras, de acuerdo con el índice IESE Cities in Motion (2016); por ejemplo, hoy se pueden destacar Smart Cities como Londres, Nueva York, Hong Kong, Barcelona, Ámsterdam y Tokio, que cuentan con aspectos fundamentales para ser eficientes, ofreciendo una alta calidad de vida. Las características que se analizan para ser consideradas como ciudades inteligentes son su modo de gobernar, la gestión pública, el medio ambiente, la proyección internacional, la cohesión social, la planificación urbana, la movilidad y transporte, el capital humano, la economía y por supuesto, la tecnología.

\section{Comunicación audiovisual en movilidad}

Las Narraciones Transmedia (NT) son aspectos indispensables, puesto que, gracias a ellas es posible comunicar lo que se desea haciendo uso de Internet en las diversas plataformas digitales y en todo tipo de medios tradicionales. Se entienden las NT como una 
forma de contar una experiencia o historia a través de diferentes soportes entrelazados por la red. Ahora bien, podemos encontrar que Carlos A. Scolari $(2014$, p. 73) habla sobre la expansión del relato de un medio a otro, teniendo en cuenta la participación de los usuarios, "Las narrativas transmedia se extienden de un extremo a otro de la ecología mediática, abarcando viejos y nuevos medios".

La primera vez que se introdujo el concepto de Narraciones Transmedia lo hizo Henry Jenkins en 2003 en su artículo Transmedia Storytelling publicado en la revista digital "Technology Review", en el cual la define como una técnica narrativa fundamentada en el desarrollo y creación de "mundos narrativos", a partir de diferentes plataformas y medios digitales, donde a su vez se integran experiencias interactivas (Jenkins, 2003). Asimismo, Jenkins (2009) explica dos características de las narraciones transmedia que se encuentran relacionadas como cuerdas que constituyen las experiencias de nuestro diario vivir y la ficción de la NT, "in immersion, then, the consumer enters into the world of the story, while in extractability, the fan takes aspects of the story away with them as resources they deploy in the spaces of their everyday life". En la primera, la explica como aquel fenómeno por el cual las personas llevan consigo aspectos de la historia que aplican en su vida, y en la segunda, se refiere a la capacidad del consumidor para acceder a mundos ficcionales.

Esta teoría es sumamente importante en la presente investigación, puesto que, a la hora de aplicarla en el caso objeto de estudio, lo que se podrá lograr es que el usuario pueda integrarse a la historia, y con esta, extraer información que le será útil. Es por ello que se hace necesario tener en cuenta la creación de contenidos específicos para cada plataforma. Una de las tecnologías que permite que el usuario experimente la inmersión es la de los videos de $360^{\circ}$, que en el caso de La Candelaria, permiten profundizar en la historia de la zona. Entonces, la narración transmedia, al ser una técnica para desarrollar de manera interactiva una historia a partir de diferentes medios y plataformas, tanto digitales como tradicionales, se puede aplicar al desarrollo de actividades culturales, y al emplear este concepto en la investigación se abre la posibilidad de contar la historia de la localidad de La Candelaria de forma fascinante, buscando como objetivo no solo la interactividad del usuario, sino también la interacción del mismo, con el consecuente disfrute de una experiencia satisfactoria teniendo en cuenta el potencial que cada plataforma ofrece.

En este punto es necesario profundizar en la diferencia entre interactividad e interacción, para evitar tropiezos en el desarrollo del trabajo de investigación. Interactividad se entiende como aquella acción que "cuando se da el control de navegación a los usuarios para que exploren a voluntad el contenido multimedia, se convierte en una navegación no-lineal e interactiva" (Vaughan, 1995, p. 230). Se comprende entonces como la capaci- dad que tiene el ser humano para hacer parte de un mensaje y al mismo tiempo contribuir con su creación; además, se caracteriza por ser un procedimiento que le proporciona al ser humano la oportunidad de comunicarse con una máquina, al tiempo que ésta le da una respuesta, teniendo en cuenta las acciones, los mensajes y el contenido que el usuario ha compartido. En relación con la interacción, O’ Sullivan (1997, p. 196) la define como "el intercambio y la negociación del sentido entre dos o más participantes situados en contextos sociales... el acento está puesto en la comunicación y la reciprocidad entre quienes promulgan, utilizan y construyen los códigos y las reglas". Entonces, es aquel intercambio entre dos o más personas en un contexto social, donde existe correlación entre ellos para construir diálogos con sentido.

Por otro lado, es necesario destacar la existencia de los tres grados de participación del usuario, que Isidro Moreno identifica como: participación selectiva, "cuando la interactividad se reduce a seleccionar entre las opciones que ofrece el programa”, participación transformativa cuando "el usuario no sólo selecciona los contenidos propuestos por el autor, sino que puede transformarlos" y participación constructiva cuando "el programa permite al usuario seleccionar, transformar e, incluso, construir nuevas propuestas que no había previsto el autor" (2012, p.9). El usuario en todo momento tiene la posibilidad de seleccionar entre muchas o pocas opciones programadas, trazando su propio camino con base en su interés.

Desde hace años se están dando cambios en la comunicación, y ahora los dispositivos móviles comparten nuestro día a día jugando un papel fundamental en nuestras vidas. Las tecnologías ofrecen la posibilidad de disfrutar de una sociedad basada en el acceso a la información virtual de manera global, intercambiando información, favoreciendo la aparición de experiencias en tiempo real gracias a estos dispositivos móviles, favoreciendo el aprendizaje y las condiciones de vida. Actualmente, debido a los medios móviles, es posible tener acceso a la geolocalización, lo que hace posible crear y desarrollar estrategias de comunicación en las que el usuario puede conectarse con un entorno cargado de información digital, para recibir datos interactivos que lo lleven a vivir una experiencia única en el lugar donde se encuentra ubicado.

Begoña Gros Salvat y Anna Forés Miravalles definen la geolocalización como aquella tecnología por la cual

Se permite asociar contenidos digitales (imágenes, vídeos, audios, etc.) a una ubicación geográfica física que puede ser activada mediante un dispositivo móvil. De este modo, el uso de este tipo de dispositivos aumenta la implicación que viene derivada del aumento de la motivación de los alumnos (2013, p. 43). 
Por lo tanto, se entiende como el medio por el cual se puede localizar geográficamente a una persona en tiempo real, independientemente de la zona en que se encuentre, siempre que se encuentre conectado a Internet. Es así como, cuando el usuario dé permiso para ser ubicado gracias al uso de la geolocalización, se le proporcionarán las coordenadas del lugar en el que se encuentra para que pueda navegar y determinar la ruta que desee utilizar, consultando, explorando y descubriendo las características del área.

Al integrar la realidad aumentada con la geolocalización, se intentará proporcionar al visitante y habitante de la localidad de La Candelaria una experiencia positiva, explorando la zona y logrando una combinación entre lo real y lo virtual. Para comprender mejor este aspecto, es necesario recurrir a Andrés Agudelo Toro, que define la realidad aumentada como:

Un tipo de ambiente virtual en el cual el usuario no se sumerge completamente en un mundo virtual sino en una mezcla de éste con el mundo real. Para el usuario aparecen los objetos virtuales y reales coexistiendo en el mismo espacio" (2005, p. 47).

Se comprende entonces, como aquella tecnología que le añade a la realidad elementos virtuales, creando así una imagen aumentada en tiempo real, que proporciona información de manera atractiva, mejorando la experiencia del usuario. Lo que se busca es experimentar con los espacios de La Candelaria permitiendo que la historia, el arte y los museos entre otros, trasciendan espacios físicos para poder contarlos de forma atractiva, teniendo en cuenta la interacción y la interactividad, a partir de la opinión de las personas que caminan por las calles de la localidad.

\section{Metodología}

La hipótesis que se plantea es que, en la ciudad de Bogotá y específicamente en la localidad de La Candelaria, dedican poca atención al desarrollo de estrategias de comunicación transmedia con el fin de comunicar su patrimonio material e inmaterial.

A partir de lo expuesto, se procede a plantear el objetivo principal de esta investigación consistente en identificar y presentar las tecnologías creadas para desarrollar y gestionar la información,TIC, y resaltar la importancia de la interactividad y la interacción, no solamente física sino también personal, para difundir de manera atractiva el patrimonio material e inmaterial de esa localidad. Esto se podrá lograr gracias a la recolección de información mediante encuestas que se realizarán a habitantes, turistas e instituciones de la localidad de La Candelaria en la ciudad de Bogotá-Colombia, para así generar en las personas interés y aprecio por descubrir la historia de la localidad de manera interactiva.
A continuación, se plantean los objetivos secundarios:

- Se pretende descubrir si los habitantes sienten que forman parte de la localidad y si son tenidos en cuenta para la creación de actividades culturales en las instituciones.

- Dado que La Candelaria es un lugar histórico, es importante descubrir qué tipo y calidad de actividades se realizan, para explorar su grado de interés, y así tener una visión clara sobre el panorama a tratar.

- Del mismo modo, se pretende identificar qué llama la atención de los turistas y habitantes que día a día caminan por las calles de la localidad así como saber cuáles son los medios que más consultan a la hora de hacer turismo y realizar actividades en la zona.

- Por último, comprobar si acuden o no a las actividades, y cuál es el grado de participación de las personas en estas.

- La finalidad de los resultados es contribuir a la creación de una visión clara sobre el estado en el que se encuentra el tema a tratar.

En este punto, lo que se quiere explicar es la manera como se pretende buscar la respuesta a la pregunta y verificar la hipótesis planteada. De acuerdo con José Cegarra Sánchez, la metodología "exige la observación, el registro de los resultados y su análisis lo más profundamente posible, siempre teniendo en cuenta la racionalidad y objetividad de la interpretación" (2004, p. 10). Entonces, es posible definirla como un conjunto de técnicas y medios lógicos que ayudan en una investigación a diseñar los problemas, sometiendo a prueba las hipótesis existentes para demostrar si son verdaderas o no.

Con la intención de desarrollar de forma clara y precisa la presente investigación, las metodologías a seguir en el presente trabajo serán exploratorias, cualitativas y cuantitativas, con el fin de ampliar el panorama sobre el tema e intentar evitar sesgos en los resultados. Adicionalmente, se pretende registrar de manera precisa los datos necesarios para partir con una base sólida en las próximas investigaciones.

Con relación a la investigación cuantitativa, Hernández, R., Fernández, C. \& Baptista, P. (2006) afirman que:

La investigación cuantitativa ofrece la posibilidad de generalizar los resultados más ampliamente, otorga control sobre los fenómenos, así como un punto de vista sobre el conteo y magnitudes de éstos. Asimismo, brinda una gran posibilidad de réplica 
y un enfoque sobre puntos específicos de dichos fenómenos, además de facilitar la comparación entre estudios similares (p. 21).

Para obtener resultados sobre uno o varios grupos de sujetos a gran escala, los estudios cuantitativos son la opción más recomendada y precisa, al permitir comparar la información obtenida de manera más fácil. Por otra parte, el objetivo de la investigación cualitativa es buscar la subjetividad, para comprender y explicar las situaciones que viven la o las personas a las que se les investiga, siendo un medio explicativo y descriptivo, que permite a la gente expresarse libremente sobre lo que sienten y sus necesidades de manera legítima (Álvarez-Gayou Jurgenson, 2003, p. 17).

Del mismo modo, Strauss y Corbin (2002, p. 21) definen tres elementos indispensables que son: los datos que se obtienen por diversas fuentes, los procedimientos que se usan para estructurar y analizar los datos y los informes escritos y verbales que se plasman en artículos, charlas o libros.

La técnica de investigación utilizada fue la encuesta, que a partir de la valoración y opinión del público a quien se le aplica, facilita una muestra de información sobre el asunto, además de brindar información fiable, cuyas respuestas pueden ser comparables.

De acuerdo con Mํa Ángeles Cea (1998, p.240) la encuesta posee cinco características que la definen: la primera hace referencia a que la información se obtiene a partir de la observación indirecta gracias a las respuestas que dan los encuestados oralmente, la segunda se refiere a que la información obtenida comprende varias cuestiones donde es posible incluir aspectos tanto subjetivos como objetivos, la tercera es la comparación de las respuestas, que se da cuando la información se recolecta de manera estructurada, la cuarta trata sobre el agrupar y cuantificar las respuestas para luego, a partir de técnicas de análisis estadísticos, examinar y relacionar entre ellas, y finalmente, la quinta se relaciona con que la significatividad de la información obtenida dependerá de los errores de muestreo y otros ajenos a este. Entonces, la encuesta se utiliza como técnica para recoger datos y así poder comparar las respuestas, y es esta acción la que permitirá analizar la información de la presente investigación de manera más profunda y de manera cualitativa. Gracias a las encuestas se podrán reunir diferentes percepciones y opiniones de forma puntual, aspecto que beneficia este trabajo.

Con relación a la muestra, según Hernández et al (2006, p.236) "para el proceso cuantitativo la muestra es un subgrupo de la población de interés (sobre el cual se recolectarán datos, y que tiene que definirse o delimitarse de antemano con precisión), este deberá ser representativo de la población". En este proyecto investigativo la selección de la muestra se realizará teniendo en cuenta tres grupos para ser encuestados: Los vecinos que habitan la localidad, visitantes, e instituciones que se encuentran ubicadas en la misma.

- Los habitantes de la Candelaria son indispensables a la hora de realizar esta investigación, puesto que ellos son quienes día a día cruzan las calles y conocen de primera mano la historia que hay en cada rincón.

- Los turistas o visitantes también son un público importante, pues aportaan desde otra perspectiva -siendo ajenos al lugar- información que los habitantes y las instituciones no brindan.

- Finalmente, las instituciones son de gran importancia, dado que en su mayoría son quienes realizan actividades culturales y apoyan la divulgación de cultura.

\section{Desarrollo de la investigación}

Como se mencionó en el punto anterior, los grupos identificados son: vecinos de la localidad, visitantes o turistas, y las instituciones. A cada grupo se le realizaron diferentes encuestas teniendo en cuenta cada una de sus características y la información que se pretendía obtener. El número de encuestas realizadas a los habitantes fue de 50, a los visitantes igualmente otras 50 y solamente se obtuvieron 15 de las instituciones, reuniendo así un total de 115 encuestas. Se es consciente que la muestra en una investigación debe ser más amplia que la ya mencionada, sin embargo, se ha realizado un gran esfuerzo para obtener los resultados teniendo en cuenta la distancia y el tiempo; además, la información obtenida puede ser orientativa para una futura investigación más profunda.

Las modalidades de encuesta utilizadas fueron tanto presenciales como por medio de Internet. En relación con la distancia, y tomando en cuenta que el caso de estudio es la localidad de la Candelaria de Bogotá, se obtuvo ayuda de terceros para aplicarlas presencialmente en el lugar establecido. En el primer grupo, de habitantes de la zona, se realizaron digitalmente, utilizando los formularios de Google, gracias a los grupos de vecinos y habitantes que se encontraron en la red social de Facebook. A falta de nueve respuestas, se complementaron presencialmente. Con el segundo grupo, instituciones de la localidad, previamente se enviaron a los respectivos correos electrónicos conseguidos en sus sitios web y demás medios digitales, obteniendo solamente tres respuestas, por lo que fue necesario ir directamente a cada una con las encuestas físicamente, que posteriormente se digitalizaron, alcanzando la meta de 15. Finalmente, en ell grupo de turistas, las 50 encuestas también se realizaron de manera presencial en la localidad. 


\section{Análisis de resultados cualitativos y cuantitativos}

\section{Resultados habitantes de La Candelaria}

Los resultados de las encuestas brindan datos cuantitativos, lo que permite abordar de una manera más aproximada el tema. Empezaremos con los resultados obtenidos del grupo de residentes de la localidad. Es posible observar que más de la mitad de los habitantes se sienten orgullosos de poder vivir en La Candelaria con un 56\%, al 30\% le es indiferente, solamente el $12 \%$ se sienten muy orgullosos, mientras que el $2 \%$ de los encuestados dicen sentirse poco orgullosos de pertenecer a dicha localidad.

De igual forma, se midió la importancia de la cultura de la localidad, mostrando que el $54 \%$ la considera importante, seguido con un $28 \%$ que considera La Candelaria como un lugar muy importante, y solamente al $18 \%$ le es indiferente. Se obtuvo un porcentaje de 0 en las calificaciones de poco importante y nada importante.

En el intento por conocer más sobre las actividades, se pidió calificar la oferta de las actividades en la localidad, a lo cual la mayoría respondió "normal" con un 54\%, el 28\% la calificó como poco buena, un 10\% como buena, el 6\% muy buena, y finalmente el $2 \%$ como nada buena. Continuando con la ruta de la pregunta, el 54\% considera que la calidad de las actividades es normal, seguido con un $18 \%$ que la considera como buena, sin embargo, se puede observar que un $16 \%$ la califican como mala, un $8 \%$ pésima y solamente un $4 \%$ cree que es de excelente calidad.

Para poder tener una visión más clara sobre la oferta y la calidad de las actividades, se deseaba saber si los habitantes participan en las diferentes actividades culturales. En este punto, más de la mitad de los encuestados respondieron con un no (74\%), un 18\% con un poco de participación y finalmente un 8\% sí asiste. Por otra parte, se preguntó si ellos consideran que la alcaldía, la localidad y las instituciones apoyan la creación de actividades culturales, y aquí prima el $52 \%$ que no sabe ni tiene conocimiento sobre este punto, contrastado con un $26 \%$ que sí lo considera y por último un $22 \%$ que afirma que no apoyan su creación. Asimismo, se consideró fundamental determinar si los habitantes consideraban que las actividades culturales se dirigían hacia ellos, donde en gran medida (72\%) respondió que no lo consideraban así, mientras que un $28 \%$ sí. Acto seguido, brindamos la posibilidad a los habitantes para que propusieran actividades, obteniendo muchas opciones, donde se destacan la creación de recorridos divertidos que atraigan la atención de las personas, al igual que la realización de vídeos 360 mostrando la historia de la localidad, también hacer actividades en las calles, la creación de videojuegos históricos y trayectos donde exista la innovación y lo virtual. Cabe mencionar que muchos habitantes consideran primordial mejorar la seguridad de la zona para realizar cualquier actividad sin preocupación. Adicionalmente, se descubrió que el 82\% de los encuestados cuenta con teléfono celular (móvil) con acceso a Internet y el 18\%, no.

Algo fundamental para esta investigación es averiguar si los habitantes conocen la historia de La Candelaria, y se puede apreciar que un porcentaje la conoce poco (48\%), un $36 \%$ afirma tener conocimiento sobre la historia y un $16 \%$ no tiene. Finalmente, se preguntó que si la localidad contara con una aplicación gratuita donde se pudiera obtener información cultural, ellos la descargarían y harían uso de ella, ante lo cual el 68\% respondió sí, mientras que al 26\% le es indiferente y un 6\% no lo haría.

\section{Resultados del grupo de turistas}

Continuando con la revisión de los resultados, se pasa a exponer el grupo de los turistas que día a día visitan no solamente la ciudad de Bogotá, sino el centro histórico de la capital, La Candelaria. Para tener un panorama sobre los visitantes se quiso conocer su lugar de origen, resultando que el 38\% provenía de Europa, el 30\% de Norteamérica, seguido con un $24 \%$ de Suramérica y un $8 \%$ de Asia. No se encontraron turistas de África y Oceanía. Asimismo, se quiso conocer la ciudad o departamento de los turistas colombianos encuestados, y el resultado fue: Medellín, Pereira, Cali, Ibagué y La Guajira. Después se preguntó si tienen algún conocimiento sobre la historia de La Candelaria, al respecto, el $84 \%$ no sabe y el $16 \%$ sí. Del mismo modo, se cuestionó acerca de sus intereses cuando visitan una ciudad, un 48\% respondió que la arquitectura es lo que más les llama la atención, un $24 \%$ tiene interés en los museos, el $22 \%$ en los monumentos, el $2 \%$ en los restaurantes, un $2 \%$ en las bibliotecas y finalmente, otro $2 \%$ en otras cosas que ofrecen las ciudades. Con un $62 \%$ la gran mayoría de los turistas hacen uso de su teléfono celular (móvil) como medio y guía para recorrer y conocer los diferentes lugares turísticos de un lugar, un 32\% usa los mapas de turismo y el 6\% contrata personas especializadas como guías turísticos. Para poder confirmar el uso indispensable del teléfono celular, se ha preguntado si cuentan con acceso a Internet en Bogotá, obteniendo como resultados que el $68 \%$ de las personas entrevistadas sí tiene acceso a este servicio, mientras que un $32 \%$ no.

En cuanto a la localidad, se deseaba saber si les parece atractivo conocer la historia que representa esta zona, y el 100\% respondió afirmativamente. Igualmente, el 100\% de los turistas respondió que tienen previsto regresar a Bogotá. Por último, se les ha preguntado que si la localidad de La Candelaria contara con una aplicación gratuita donde pudieran obtener información cultural, ellos la descargarían y harían uso de ella, donde de nuevo el $100 \%$ respondió afirmativamente. 


\section{Resultados del grupo de instituciones}

Para finalizar con la presentación de los resultados de las encuestas, se expone la información obtenida en el grupo de las Instituciones. La primera pregunta realizada fue sobre si han organizado actividades culturales en la localidad. El 86,7\% sí lo ha hecho, destacándose los talleres culturales, las obras de teatro y conferencias, entre otras. Sin embargo, 13,3\% no ha realizado actividad alguna. Igualmente, se les cuestionó sobre la posibilidad de ver sus actividades publicadas en algún medio, a lo que el 93,3\% dijo que sí y el 6,7\% respondió negativamente. Los que contestaron afirmativamente mencionaron medios como las redes sociales, sus sitios web y correos masivos. En relación con la cantidad aproximada de personas que acuden a dichas actividades, se logró establecer un término de entre 20 a 200, aunque es necesario aclarar que otras instituciones con mayor capacidad, han dado como máximo 400 y 4.000 personas. Asimismo, se quería descubrir en qué medida las instituciones creen que el acervo histórico fundamental de La Candelaria se conoce, a lo que el 53,3\% indicó que poco, contra un $46,7 \%$ que afirmó que se sabe mucho.

Por otro lado, era necesario saber si las instituciones consideran a los habitantes de la localidad como público importante para las actividades culturales que realizan. El 86,7\% afirmó tener en cuenta a los vecinos, sin embargo, el 13,3\% no lo hace. Se preguntó por la percepción acerca del uso de teléfonos celulares como medio para difundir la cultura de un lugar, a lo que la mayoría, con un 33,3\% respondió muy importante, seguido de un $26,7 \%$ que lo considera importante, un 26,7\% lo considera normal; por último, el 13,3\% de las instituciones opinaron que es poco importante. Complementando la anterior pregunta, la gran mayoría consideró que el uso de dispositivos móviles como medio para comunicar la cultura de la localidad es muy efectivo, relevante e importante, puesto que actualmente es un medio con el que las personas pasan mucho tiempo.

Se les preguntó si consideran que los turistas y habitantes acceden de manera fácil y ágil a las diferentes actividades culturales en La Candelaria, primando el sí con un 86,7\% sobre un 13,3\% que respondió negativamente. De igual forma, el 60\% de las instituciones sî tienen en cuenta a los turistas a la hora de crear y desarrollar actividades culturales, frente a un $40 \%$ que no lo hace. Aquellas que respondieron afirmativamente explicaron que lo hacen mediante la difusión en el sector, también por medio de recorridos y visitas guiadas, además con la difusión de información en las embajadas, en los medios de comunicación y en las redes sociales.

Finalmente, se les preguntó sí tienen previsto desarrollar aplicaciones para celular sobre la localidad, a lo que el $60 \%$ respondió negativamente, contra un $40 \%$ de respuestas positivas. Aquellas que respondieron sí, se les cuestionó en qué sentido y a qué público estaría dirigida la aplicación, mas no dieron respuesta alguna, dejando en evidencia la poca claridad que tienen sobre el asunto.

\section{Justificación de verificación de la hipótesis}

Durante la recolección de los resultados y su análisis posterior, se obtuvo información que verifica la hipótesis planteada, que en este punto se considera necesario recordar: "en la localidad de La Candelaria de la ciudad de Bogotá, dedican poca atención al desarrollo de estrategias de comunicación transmedia con el fin de comunicar su patrimonio material e inmaterial". En las entrevistas realizadas se reunió todo tipo de información que sin duda abre paso a más vías de investigación, además, se consiguieron una serie de evidencias que convalidan la hipótesis planteada.

Los resultados obtenidos hacen mención a que las actividades culturales que se realizan en la localidad son de carácter normal tanto en calidad como en oferta, deseando poder ver innovación en estas, lo que sugiere la poca o casi nula existencia de estrategias transmedia, dejando un vacío para los turistas, quienes hacen más uso de dispositivos móviles con acceso a Internet para descubrir una ciudad, algo que sin duda puede ser aprovechado para el bien de La Candelaria.

\section{Interpretación de resultados}

Los resultados obtenidos a partir de las 115 encuestas realizadas serán interpretados de acuerdo con la categoría de las preguntas, reuniendo los tres grupos.

Los residentes de La Candelaria se identifican con la Localidad, arguyendo sentirse orgullosos de vivir en esa zona, y resaltan la importancia cultural que tiene la localidad en la ciudad de Bogotá, donde la gran mayoría cree que la oferta de actividades culturales y su calidad son de estándar normal, si bien es posible percibir que resalta la poca conformidad de muchas personas en relación con los dos últimos puntos. Del mismo modo, las instituciones que respondieron la encuesta afirmaron que han creado actividades en la localidad, que se pueden encontrar en sus páginas web y redes sociales. Junto con ello, declaran una asistencia aproximada de 20 a 400 de personas a estas actividades, claramente dependiendo de la institución e instalaciones de la misma; si bien la mayoría de los residentes manifiestan no participar de ellas; no obstante, no tienen pleno conocimiento de si la alcaldía y otras instituciones participan en la elaboración de estas.

Continuando con las actividades culturales, los residentes creen que hasta el momento no han sido tenidos en cuenta para la realización de estas, sin embargo, contrasta con la opi- 
nión de las instituciones, que afirman que sí son considerados como público importante. En comparación con la importancia de los turistas, la visión de las instituciones entrevistadas casi se divide, ya que solamente un poco más de la mitad afirma que sí son tenidos en cuenta a través de las embajadas y por medio de la divulgación de información. Con relación a la historia de La Candelaria, claramente se puede observar que los turistas no tienen ningún conocimiento de ésta, lo que es entendible; no obstante, un poco menos de la mitad de los habitantes entrevistados afirman conocer solamente un poco los hechos históricos, siendo la más alta respuesta. Lo anterior se ratifica con las respuestas dadas por las instituciones, ya que la mayoría afirma que poco se conoce sobre el tema. Al analizar, se percibe un preocupante aspecto, y es que la mayoría de los vecinos que diariamente caminan por el centro histórico de Bogotá, solamente saben un poco acerca del lugar donde viven, y por ello se les ha pedido sugerir actividades, a lo que propusieron realizar recorridos históricos diferentes a los existentes con ayuda de lo virtual, además de implementar aspectos innovadores con apoyo de tecnologías como los videos $360^{\circ}$ y crear videojuegos sobre la historia de la zona, entre otros

En relación con el uso de dispositivos móviles, la mayoría de los residentes y turistas afirmaron contar en la ciudad con acceso a Internet en sus celulares, así mismo, expresaron que si La Candelaria contara con una aplicación gratuita sobre información histórica y cultural todos la descargarían y harían uso de ella. Entonces, es posible observar que un gran porcentaje de los visitantes hacen uso de dispositivos móviles como guía en una ciudad para conocer las actividades que se ofrecen, dejando en segundo plano a los mapas y guías turísticos. Por lo tanto, existe un gran uso de esta tecnología que puede ser aprovechado.

Por otro lado, las instituciones perciben el uso de celulares como medio importante, relevante y efectivo para comunicar información cultural de un lugar. Sin embargo, no todas tienen previsto desarrollar aplicaciones, y quienes en un futuro lo harían no tienen una visión clara sobre cómo sería ni para qué público se crearía. Los turistas llegan con el fin de descubrir y conocer todo lo que ese lugar les ofrece, aun así, en los resultados obtenidos se determinó que ellos fijan su atención primordialmente en la arquitectura que La Candelaria les ofrece. Finalmente, los viajeros afirman que tienen previsto visitar de nuevo Bogotá.

\section{Conclusiones}

Esta investigación se ha realizado con el propósito de obtener información sobre la manera como actualmente se comunica a las personas el patrimonio cultural material e inmaterial de La Candelaria, y la percepción de ellos en relación con las actividades ofrecidas. De acuerdo con la información recolectada y lo discutido anteriormente se pueden establecer las siguientes conclusiones sobre la investigación:
- Se identificó que los habitantes se sienten felices de poder vivir en una zona con tanta historia, sin embargo, no se sienten totalmente considerados a la hora de desarrollar actividades culturales, algo que sin duda se ve reflejado en su poca participación. Esto sin duda es importante, puesto que la base para crear estrategias de comunicación transmedia efectivas es que los habitantes se sientan parte de La Candelaria y orgullosos de poder hacer parte de ella.

- Por otra parte, sabiendo que tanto habitantes como turistas cuentan con un dispositivo móvil con acceso a internet, se abre la puerta a un posible desarrollo de aplicaciones que permitan comunicar su patrimonio material e inmaterial, oportunidad que no se encuentra establecida actualmente como futura meta por parte de las instituciones, quienes afirman querer desarrollar una aplicación aunque no han decidido a qué público iría dirigida ni cuál sería su uso.

- Además, no hay interactividad ni interacción en la localidad con su patrimonio material e inmaterial, por ende, claramente no existe proceso alguno de inmersión.

Los resultados obtenidos en este trabajo permiten afirmar que no existe ninguna estrategia de comunicación transmedia en el ámbito cultural e histórico para los habitantes y visitantes, algo que podría mejorar el turismo y a su vez resguardar la historia, y sitúa en esta materia a Bogotá por debajo de otras ciudades.

A partir del presente trabajo y con la información obtenida, surgen nuevas cuestiones para investigar y nuevas hipótesis para contrastar, cumpliendo con la finalidad del estudio, que era que, a partir de los resultados obtenidos, se pudiera contribuir a la creación de una visión clara sobre el estado en el que se encuentra el tema tratado.

La localidad de La Candelaria, al ser reconocida como el centro histórico de Bogotá, garantiza el aprovechamiento y la creación de muchas opciones de actividades, y por ende, se pueden desarrollar y aplicar estrategias transmedia que mejoren su calidad. Se abre una oportunidad para generar estrategias de comunicación transmedia para que todas las personas que viven y visitan la localidad puedan sentirse parte de ella; esto se podrá lograr partiendo como base de la interacción y la interactividad, para así crear actividades pensadas en el disfrute del patrimonio material e inmaterial de la localidad, fortaleciendo la experiencia del usuario. Sobre los grupos seleccionados, se propone continuar con el estudio del tema con ellos, realizando entrevistas en profundidad y encuestas con una muestra representativa, para obtener así resultados importantes y con un alto grado de confiabilidad. 
Ahora bien, ha surgido una serie de hipótesis plausibles a lo largo del desarrollo del trabajo, que son:

- La historia de la localidad de La Candelaria no está comunicando la esencia en su totalidad.

- Se está perdiendo la identidad cultural por la poca representatividad de las prácticas comunicativas en los acontecimientos históricos de la zona.

- $\quad$ La narración con la que cuenta la localidad es básica y limitada, no existen múltiples miradas ni formas de transmitirla.

- Los habitantes de La Candelaria conocen la importancia que tiene su localidad por los hechos históricos aprendidos en las aulas, más no por las actividades culturales que se realizan.

- La comunicación transmedia es poco considerada a la hora de realizar estrategias para el desarrollo cultural de la zona.

- $\quad$ Este trabajo es una base, un primer paso, que precede a la continuación del estudio sobre el tema, lo que abre nuevas líneas de investigación que son:

- $\quad$ Realizar entrevistas en profundidad tanto a habitantes de la localidad como a instituciones, con preguntas semiestructuradas para reunir datos más detallados sobre el patrimonio cultural material e inmaterial.

- Estudiar, analizar y crear estrategias a partir de los medios de comunicación digitales convergentes en La Candelaria para no dejar en el olvido su historia.

- Definir qué nuevas formas de producción audiovisual son las adecuadas para comunicar el patrimonio material e inmaterial y así conservarlo.

Se espera la continuación de la investigación, este trabajo es un primer paso hacia nuevas líneas, la localidad es indispensable en la capital de Colombia, tanto por su historia como por el turismo que atrae, y sin duda abre la oportunidad para la creación de estrategias que beneficien a La Candelaria.

\section{Referencias}

Agudelo, A. (19 de enero de 2005). Modelo de contexto para realidad aumentada. Revista Universidad EAFIT. Vol. 41. No. 13B. Pp. 44-64. Recuperado de http://goo.gl/y9ouaF Alcaldía Mayor de Bogotá (Sin fecha). Secretaría General. Recuperado de http://goo.gl/ WOQM68

Álvarez-Gayou Jurgenson, J. L. (2003). Cómo hacer investigación cualitativa. México: Paidós. Ausubel, D., Novak, J.

Berrone, P. y Ricart, J. (2017). IESE Cities in Motion Index. Recuperado de https://goo.gl/ MHLsbm

Bustillo, E., y Rodríguez, P. (2015). Los sistemas de información geográfica y las ciudades inteligentes. Polígonos Revista de geográfica. Recuperado de http://goo.gl/YJ0Bju

Cea D’Ancona, M. a Ángeles (1998). Metodología cuantitativa: estrategias y técnicas de investigación social. Madrid: Editor Síntesis.

Estebanell, M. (2002). Interactividad e Interacción. Revista LatinoAmericana de Tecnología Educativa. Volumen 1. número 1. Recuperado de http://goo.gl/LssyY

Freitas, C. y Castro, C. (2010). «Narrativas audiovisuales y tecnologías interactivas». Revista Estudios Culturales. Recuperado de http://goo.gl/jcpVav

Gros, B., y Forés, A. (2013). El uso de la geolocalización en educación secundaria para la mejora del aprendizaje situado: Análisis de dos estudios de caso. RELATEC Revista Latinoamericana de Tecnología Educativa. Vol. 12 (2) 41-53. Recuperado de http://goo. $\mathrm{gl} / 3 \mathrm{dK} 9 \mathrm{xO}$

Hernández, R., Fernández, C. \& Baptista, P. (2006). Metodología de la Investigación. México: Mc Graw Hill.

IESE Cities in Motion (2016). Índice IESE Cities in Motion. Recuperado de http://goo.gl/ kvzKwk

leseinsight (2016). IESE Insight Londres encabeza el ranking de las ciudades más “inteligentes". Recuperado de http://goo.gl/B8h1iC

Ito, T. (2006). Arquitectura de límites difusos. Barcelona: Gustavo Gili.

Jenkins, H. (Enero 15 de 2003). Transmedia Storytelling. Moving characters from books to films to video games can make them stronger and more compelling. Revista MIT Technology Review. Recuperado de http://goo.gl/bssAvh

enkins, H. (Diciembre 12 de 2009). Revenge of the Origami Unicorn: Seven Principles of Transmedia Storytelling. En Aca-Fan. The official weblog of $\mathrm{H}$.

Jenkins. Recuperado de (parte 1) http://goo.gl/DncSCY

y (parte 2) http://goo.gl/NR38MR

Moreno, I. (2012). Narrativa hipermedia y transmedia. Recuperado de http://goo.gl/aRqOU1 Moreno, I. (2014). Interactividad, interacción y accesibilidad en el museo transmedia. Recuperado de http://goo.gl/8NmrJZ 